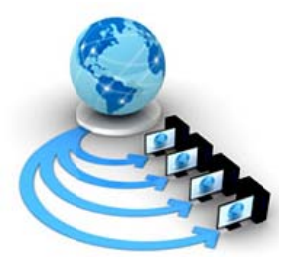

Volume 9, No. 1, January-February 2018

International Journal of Advanced Research in Computer Science

REVIEW ARTICLE

\author{
Available Online at www.ijarcs.info
}

\title{
A REVIEW ON DIFFERENT UNDERWATER IMAGE ENHANCEMENT APPROACHES
}

\author{
Kuldeep Kaur \\ Student of Master in Technology \\ Department of Computer Science and Application \\ Chaudhary Devi Lal University, Sirsa, Haryana, India
}

\author{
Monika Bansal \\ Assistant Professor \\ Department of Computer Science and Application \\ Chaudhary Devi Lal University, Sirsa, Haryana, India
}

\begin{abstract}
In this review paper, different underwater image enhancement techniques are studied. Underwater imaging is a challenging in the zone of photography specifically for low resolution and also the ordinary digital camera. Because of there are some issues occur in underwater pictures like as low contrast, blurring, limited range visibility, noise, non-uniform lighting, color diminished, and bright artifacts so due to this reason the underwater picture is not clear. Hence in the underwater image enhancement, the main task is to improve the image visibility and restore the color by eliminating unwanted effect during the processing of imaging.
\end{abstract}

Keywords: Underwater image, blurring, noise, visibility.

\section{INTRODUCTION}

In these days underwater imaging is a vast application and becomes important investigation field because the rivers, lakes, and seas include many valuable resources inside it. But, the difficulty with the underwater is the loss of color and also the contrast of the picture since according to the significant depth the color and the contrast of the underwater picture is a change [1]. The reflection of the light varies greatly with respect to the light reflection that depends on the configuration of the oceans. Another concern is associated with the water that curves the light either to make crinkle patterns or to diffuse. Most essentially the variety of the water controls and effect the refine properties of the water such as trickle of the dust in the water. The light is partly entered in the water vertically and partly polarized horizontally according to the reflected amount.

An essential nature of the vertical polarization is that it makes the protest less sparkling and catches profound color which may not be conceivable to catch generally. Another outstanding issue related to the underwater pictures is a concern with the thickness of the water in the ocean which is viewed as 800 times denser medium than air. Thusly, when light rays transfer from the air to the water, it is somewhat reflected invert and in the meantime halfway enters the water. The aggregate light sum that enters the water likewise begins lessening begin it goes further in the ocean. Likewise, the water molecules to absorb a certain quantity of light. Thus, the underwater pictures are obtained darker and darker as the profundity increments. Not just the amount of light rays is consolidated when it goes further yet in addition color drop off one by one relying upon the wavelength of the color. For instance, above all else, red color vanishes at the profundity of $3 \mathrm{~m}$. Besides, orange color begins vanishing while we go further. At the profundity of $5 \mathrm{~m}$, the orange color is lost. Thirdly a large portion of the yellow color goes off at the profundity of $10 \mathrm{~m}$ subsequently the purple and green dissipate at father profundity [12].

Different Underwater image enhancement techniques used for recovery of distorted underwater images:

(1) Dark-channel prior method (DCP): To restore original clearness of the underwater image a dark channel method is an efficient and effective method. Because of the light attenuation, the picture taken in the underwater environment is distorted. On the statistics of the clear picture in the air, the DCP is based. It is very often that some pixels have very less intensity in pure water. These pixels are called dark pixels. By the background light, the intensity of these dark pixels is contributed in the underwater images. Therefore the accurate estimation of water transmission is provided by the dark pixels. For the high-quality water free picture and produce a good depth map, this method can be used. Even in the heavy blur images this approach is physically feasible and can obtain the distant objects. When scene object is inherently homogeneous to the background light over a huge local region the DCP may not be useful.

(2) WCID Technique: The WCID is called Wavelength Compensation and Image Dehazing. The WCID is the technique or method underwater image enhancement which compensate wavelength. A WCID depends on an underwater image formation model. The calculation of wavelength pay and picture dehazing joins systems to expel twisting caused by color change and light scattering. First, Dark channel prior technique is utilized to gauge remove amongst camera and protest in view of the profundity delineate, the frontal area and foundation ranges are portioned. The light forces of forefront and foundation are contrasted with deciding nearness of simulated light if fake light source is recognized, the luminance presented by it is expelled from the closer view territory. Next dehazing calculation is utilized to evacuate cloudiness impact and color change. 
(3) Histogram equalization: Histogram equalization is a picture processing strategy which utilizes picture histogram for picture contrast change. This strategy, for the most part, builds the contrast of distorted (contorted) image by modifying powers. The technique is valuable in images with foundation and closer views that are both splendid or both dim. Histogram equalization is a strategy for altering image forces to upgrade contrast [11].

In the second section we studied the literature review of the previous papers and further discussed the literature review table. In the third section conclusion is discussed.

\section{LITERATURE REVIEW}

Sudhansu Mallik et. al. [1]: In this paper, they introduce Contrast Limited Adaptive Histogram Equalization (CLAHE) color model for haze removal and their main focus on the underwater picture improvement through haze removal algorithm by using DCP (Dark channel prior) strategy. It displays a good output by reducing noise and haze effect but in some circumstance, it darkens the picture. Histogram Equalization (HE) technique used to enhance the dehazing output. Contrast Limited Adaptive Histogram Equalization on RGB model has been followed in our way to deal with change the level of contrast and force of dehaze picture. At last, a color correction algorithm for the visually appreciable result has been used.

Tao Chen et. al. [2]: In t his paper, Tri-state median filter is used to maintain the picture detail and also used for repressing impulse noise. Before applying filter thoroughly, they integrate the center weighted median filter and the standard median filter into a noise detection configuration to find whether a pixel is damaged. Extensive simulation outputs exhibit that the planned filter constantly better than other

Amir Beck et. al. [3]: In this article analyze gradient-based strategy for picture deblurring and denoising issue based on the discretized Total Variation (TV) reduction model with restriction. They obtain a quick algorithm for the constrained total variation -based picture deburring issue. To attain this task they integrate an acceleration of the widelyknown double perspective to the denoising issue with a unique monotonous variant of a "Fast Iterative Shrinkage/Thresholding Algorithm (FISTA)" they have currently established. The outcome Gradient-Based algorithm portion is a significant simply joint with a demonstrate the global rate of confluence which is significantly more appropriate than presently known gradient projections-based techniques. Their outcomes are relevant to both the isotropic and anisotropic discretized Total Variation functional.

Alin Achim et. al. [4]: Synthetic aperture radar pictures are basically pretentious by multiplicative speckle noise, which is the cause of the rational nature of the scattering phenomenon. In this article proposed a novel Bayesianbased algorithm within the framework of wavelet examine, which minimize speckle in Synthetic aperture radar picture although preventing the structural property and textural detail of the scene. Firstly, they exhibit that the subband decompositions of logarithmically changed Synthetic aperture radar picture are correctly modeled by alpha-stable distributions, a class of heavy-tailed densities. They utilize the alpha-stable model to build up a blind speckle- suppression processor that implements a nonlinear operation on the data and they compare this nonlinearity to the degree of non-Gaussianity of the data. In conclusion, they evaluate their anticipated technique to current state-of-the-art soft thresholding method applied to real Synthetic aperture radar imagery and they estimate the obtain presentation enhancement.

D. Darian Muresan et. al. [5]: This paper shows a novel perspective to picture denoising utilizing adaptive principal apparatus. Their estimations are that the picture is corrupted by additive white Gaussian noise. The new denoising method implements good in terms of PSNR values and in terms of picture visual fidelity, the new method differentiates very well averse to a few of the most currently declared denoising algorithm.

Frédéric Petit et. al. [6]: In this article, an underwater image enhancement technique utilizing quaternions is exhibited. This work expects to enhance the color performance and contrast of the articles as if the scene has been removed from the water. This technique depends on light disable reversal after operate a color space contraction utilizing quaternions. Connected to the white, the lessening gives a hue vector characterizing the watercolor. Utilizing this reference pivot, geometrical transformations into the color space are figured with quaternions. Pixels of water regions of prepared pictures are processed to gray or colors with a low immersion while the items stay completely colored. So in this manner, the contrast of the watched scene is essentially made strides improved, the contrast between the foundation and whatever is left of the image is expanded, giving the first approach towards a presegmentation step.

Haocheng Wen et. al. [7]: In this paper, they determine another underwater optical model to depict the development of an underwater picture, and at that point propose a compelling underwater picture improvement calculation with this model. Their calculation is compelling and physical legitimate, and can well handle deep-sea picture and these pictures caught from turbid waters.

Balvant Singh et. al. [8]: Image enhancement is an approach to enhancing the quality of the picture by enhancing its feature. Because of the poor detectable condition, the underwater picture suffers from low contrast and resolution, hence an object recognition becomes the typical task. This article comparative study of different contrast enhancement method for such underwater picture is shown. Contrast stretching, and histogram equalization (HE ) method compare with the performance of Contrast Limited Adaptive Histogram Equalization(CLAHE) method. SNR and Mean square Error (MSE) parameter are used to comparison the performance of underwater image.

Rajesh Kumar Rai et. al. [9]: In the underwater pictures the objects are not clearly observable because of the scattering of light and low contrast and the large noise exist in the underwater area. So it is hard to segmentation in such area without missing the detail of the object. In this article, a process of segmentation is executed for underwater pictures, in which the image quality is primarily improved using contrast limited adaptive histogram equalization method and later histogram thresholding is using to segment the items. And also consider the comparative analysis of the different Histogram thresholding methods. SNR and Mean square 
Error (MSE) parameter are used to comparison the performance of underwater image.

Kashif Iqbal et. al. [10]: In case of underwater, transparency of pictures is corrupted by light retention and dispersing. This reason one shading to require the picture. So as to enhance the discernment of underwater pictures, they suggest an approach in view of slide stretching. The goal of this approach is twofold. Furthermore, the intensity and saturation stretching of HSI is utilized to expand the real nature and tackle the issue of lighting. Interactive software has been created for underwater picture improvement. Outcomes of the software are shown in this paper.

Table 1: A brief overview of this literature review

\begin{tabular}{|l|l|l|l|}
\hline Author Name & Year & Technology Used & Result \\
\hline Tao Chen et. al. & 1999 & Tri-state median filter & Good Visual quality \\
\hline Alin Achim et. al. & 2003 & Bayesian wavelet shrinkage & Noiseless and better visual quality \\
\hline Balvant Singh et. al. & 2011 & CLAHE method & Improve the contrast of image \\
\hline Rajesh kumar Rai et. al. & 2012 & $\begin{array}{l}\text { CLAHE enhancement and } \\
\text { thresholding and also }\end{array}$ & $\begin{array}{l}\text { Better equalization and } \\
\text { improves the contrast of image }\end{array}$ \\
\hline Kashif Iqbal et. al. & 2007 & An Integrated Colour Model & Enhance underwater image \\
\hline
\end{tabular}

\section{CONCLUSION}

In this paper, we study the different underwater image enhancement methods for underwater image and also study the different issues that occur in the underwater image like as low contrast, blurring, poor visibility, noise, color diminished, and non-uniform lighting. Different underwater image enhancement techniques used to improve the quality, contrast of the picture and remove the different noise particles.

\section{REFERENCES}

[1] Sudhansu Malik, Salman S.Khan, and Umesh C. Pati. "Underwater Image Enhancement Based on Dark Channel Prior and Histogram Equalization." .2016 International Conference on Innovations Embedded and Communication System(ICIIECS'16).

[2] Tao Chen, Kai-Kuang Ma, and Li-Hui Chen. "Tri-state Median Filter for image denoising." IEEE Transactions on Image Processing, vol. 8, no. 12, December 1999: 18341838.

[3] Amir Beck, and Marc Teboulle. "Fast Gradient-Based algorithms for constrained total variation image denoising and deblurring problems." IEEE Transactions on Image Processing vol. 18, no.11, Nov. 2009 : 2419-2434.

[4] Alin Achim, Panagiotis Tsakalides, and Anastasios Bezerianos. "SAR image denoising via Bayesian wavelet shrinkage based on heavy-tailed modeling." IEEE Transactions on Geoscience and Remote Sensing vol. 41, no.8, August 2003: 1773-1784.
[5] D. Darian Muresan, and Thomas W. Parks. "Adaptive principal components and image denoising." International Conference on Image Processing ,2003. ICIP 2003.

[6] Frédéric Petit, Anne-Sophie Capelle-Laizé, and Philippe Carré. "Underwater image enhancement by attenuation inversion with quaternions." IEEE International Conference on Acoustics, Speech, and Signal Processing (ICASSP). 2009.

[7] Haocheng Wen, Yonghong Tian, Tiejun Huang and Wen Gao. "Single underwater image enhancement with a new optical model." IEEE International Symposium on Circuits and Systems (ISCAS), 2013. IEEE, 2013.

[8] Balvant Singh, Ravi Shankar Mishra, and Puran Gour. "Analysis of contrast enhancement techniques for underwater image." International Journal of Computer Technology and Electronics Engineering vol.1 ,no.2 , 2011: 190-194.

[9] Rajesh Kumar Rai, Puran Gour, and Balvant Singh. "Underwater image segmentation using CLAHE enhancement and thresholding." International Journal of Emerging Technology and Advanced Engineering vol. 2, no. 1, January 2012: 118-123.

[10] Kashif Iqbal, Rosalina Abdul Salam, Azam Osman and Abdullahh Zawawi Talib "Underwater Image Enhancement Using An Integrated Colour Model." IAENG International Journal of Computer Science 34.2 (2007): 239-244.

[11] Bhanudas Sandbhor and G. U. Kharat. "A Review on Underwater Image Enhancement Techniques." International Journal of Advanced Research in Computer Science and Software Engineering vol.5, no. 5, May2015.

[12] Pooja Sahu, Neelesh Gupta, and Neetu Sharma. "A survey on underwater image enhancement techniques." International Journal of Computer Applications vol.87, no.13, February 2014. 